\title{
Trauma and depressive symptomatology in middle- aged persons at high risk of dementia: the PREVENT Dementia Study
}

\author{
Karen Ritchie (1) 1,2,3 Isabelle Carrière, ${ }^{1}$ Sarah Gregory, ${ }^{2}$ Tam Watermeyer, ${ }^{2,4}$ \\ Samuel Danso, ${ }^{2}$ Li Su, ${ }_{1}^{5}$ Craig W Ritchie, ${ }^{2}$ John T O'Brien (1) ${ }^{5}$
}

${ }^{1}$ U1061 Neuropsychiatry, INSERM, University of

Montpellier, Montpellier, France ${ }^{2}$ Centre for Dementia

Prevention, Centre for Clinical Brain Sciences, University of Edinburgh, Edinburgh, UK ${ }^{3}$ Institut du Cerveau, Paris, France

${ }^{4}$ Department of Psychology, Northumbria University, Newcastle upon Tyne, UK ${ }^{5}$ Department of Psychiatry, University of Cambridge, Cambridge, UK

\section{Correspondence to} Prof Karen Ritchie, U1061 Neuropsychiatry, INSERM University of Montpellier, Montpellier, France 34093; karen.ritchie@inserm.fr

$\mathrm{KR}$ and IC are joint first authors.

Received 11 May 2020 Revised 23 September 2020

Accepted 23 September 2020 Published Online First 21 October 2020

\section{ABSTRACT \\ Objective Depression and trauma are associated} with changes in brain regions implicated in Alzheimer's disease. The present study examined associations between childhood trauma, depression, adult cognitive functioning and risk of dementia.

Methods Data from 378 participants in the PREVENT Dementia Study aged 40-59 years. Linear and logistic models were used to assess associations between childhood trauma, depression, dementia risk, cognitive test scores and hippocampal volume.

Results Childhood trauma was associated with depression and reduced hippocampal volume but not current cognitive function or dementia risk. Poorer performance on a delayed face/name recall task was associated with depression. Childhood trauma was associated with lower hippocampal volume however poorer cognitive performance was mediated by depression rather than structural brain differences. Conclusion Depressive symptomatology may be associated with dementia risk via multiple pathways, and future studies should consider subtypes of depressive symptomatology when examining its relationship to dementia.

\section{INTRODUCTION}

Alzheimer's disease (AD) and depressive disorders were once considered to be mutually exclusive; the co-occurrence of depression and cognitive decline being termed 'pseudomentia'. ${ }^{1}$ Depression is now known to be closely associated with $\mathrm{AD}$, with a prevalence around $50 \%$ at diagnosis ${ }^{2}{ }^{3}$ compared with $20 \%-25 \%$ for the general elderly population. ${ }^{4}$ A meta-analysis ${ }^{5}$ based on prospective population studies and adjusting for multiple confounders has estimated that late-life depression increases 1.65 fold the risk of future dementia, and also increases the risk of progression from mild cognitive impairment to dementia.

A central problem has been that the underlying mechanism which links the two pathologies is still uncertain, and controversy continues as to whether depression is a risk factor for $\mathrm{AD}$ or a prodromal feature of the disease itself. There is strong evidence that depressive symptoms are a direct result of AD-related brain changes. Structural and functional brain changes show depression in $\mathrm{AD}$ to be related to disruption of frontal-striatal and subcortical limbic pathways, either due to grey matter (GM) loss $^{6}$ or white matter (WM) lesions ${ }^{7}$ accompanied by serotoninergic deficiency and a dopaminenorepinephrine imbalance. ${ }^{8}$ Other studies have reported associations with tau accumulation and amyloid metabolism. ${ }^{9-12}$ Several epidemiological studies further suggest that depression onset occurs mostly in the few years preceding the diagnosis of $\mathrm{AD}$ dementia, and is therefore prodromal. ${ }^{12} \mathrm{~A}$ comparison of familial $\mathrm{AD}$ mutation carriers with their non-carrier relatives found reduced depression scores in mutation carriers in the presymptomatic stages of the disease, but elevated levels in carriers once dementia symptoms emerged, suggesting depression may be a symptom of onset rather than a risk factor for $\mathrm{AD}{ }^{13}$

On the other hand a meta-analysis of prospective studies showed an inverse association with proximity to dementia, suggesting it to be a risk factor rather than a prodrome,${ }^{14}$ and a postmortem study of persons with current major depression found no association between AD neuropathology and cognitive loss ${ }^{15}$ suggestive of independent aetiological processes.

Longer term prospective studies reaching back into the preclinical phases of $\mathrm{AD}$, and the possible origin of the association, are lacking. A recent 10 -year retrospective study of clinical and biomarker changes in persons with incident $\mathrm{AD}$ observed that depressive symptomatology was significantly higher 10 years before dementia diagnosis, remaining at a stable level to up to diagnosis independently of changes in other AD markers. ${ }^{16}$ This finding suggests the lack of a dose-response effect in the association between depression and biomarker changes, and that depression may pre-date brain changes in $\mathrm{AD}$. Depression thus appears to be linked to, but not entirely explained by prodromal $\mathrm{AD}$ neurodegeneration. Moreover, depression may be the endpoint of multiple aetiological pathways implicating different underlying neuropathological processes and having different associations with $\mathrm{AD}$ onset. ${ }^{17}$ Research in this area should thus attempt to focus on more homogeneous subtypes of depression.

Neurobiological studies have demonstrated that childhood maltreatment may alter brain development by programming the glucocorticoid, noradrenergic and vasopressin stress response systems to over-react to new stressors, ${ }^{18}$ thus rendering the individual increasingly vulnerable to psychiatric 
disorder. These effects appear to be long-lasting ${ }^{19}$ inducing structural and functional changes, notably reduced development of the hippocampus and amygdala, and abnormal frontotemporal electrical activity. ${ }^{18}$ These brain structures having also been implicated in the aetiology of both depression and dementia, it is not surprising that child abuse has been associated with not only increased risk for lifetime depression ${ }^{20}{ }^{21}$ but also lower cognitive scores. ${ }^{22}$ Several small clinical studies of young adult women with a history of physical and sexual abuse reported disorders of vigilance, memory and mathematical ability compared with controls. $^{23-25}$

Depression associated with childhood trauma may thus provide a clear-cut model of the association of a depression subtype with AD.

The present study is primarily designed to determine whether the association between childhood trauma and poorer cognitive performance is directly due to trauma-related hippocampal volume or mediated by depression. It further seeks to establish whether the impact on cognition may be greater in persons at genetic risk of dementia, thereby increasing brain burden in the face of possible pre-clinical changes. A secondary aim is to develop a putative model of the associations between trauma, depression, cognitive performance and dementia risk which may guide future longitudinal studies. The study uses data from a cohort of middle-aged persons in which around half have a family history of dementia in whom preclinical hippocampal changes have already been detected. ${ }^{26}$

The following hypotheses are examined:

1. Middle-aged persons who have experienced childhood trauma will have higher rates of clinical depression than those who have not.

2. Middle-aged persons exposed to childhood trauma will have lower hippocampal volume.

3. Childhood trauma will be associated with current poorer cognitive function.

4. Cognitive performance will be poorest in persons with both a history of childhood trauma and a high dementia risk.

\section{METHODS}

Subjects were recruited from the PREVENT Dementia Study; a prospective population study enriched for dementia risk, with around half of the participants having a family history of dementia. The protocol has been described in detail elsewhere $^{27} \quad$ (http://bmjopen.bmj.com/cgi/content/full/bmjopen2012-001893). Briefly participants were recruited through the dementia register database, the Joint Dementia Research website and information about the study on the internet and in study presentations. The present study included 378 participants from west London, Oxford and Cambridge for whom baseline data were available and validated on all variables and of whom 203 have a parent with diagnosed $\mathrm{AD}$, vascular or mixed dementia. Persons with a parent with diagnosed dementia were considered to be at increased risk of future dementia and current preclinical brain changes.

\section{Trauma}

Lifetime trauma was assessed using the Life Stressor ChecklistRevised, ${ }^{28}$ a 30 -item self-administered questionnaire which assesses age of occurrence, perceived level of danger and subsequent impact on everyday life in the past year. The Diagnostic and Statistical Manual of Mental Disorders, fourth edition (DSM IV) ${ }^{29}$ criterion A for post-traumatic stress disorder (PTSD) was applied to individual responses to distinguish trauma from milder transient stressors (for example moving house) and events with low perceived level of threat. Absence of information on the presence of subsequent intrusive symptoms did not allow us to make a formal diagnosis of PTSD. The items retained in relation to criterion A were death of someone close, physical and emotional abuse, sexual abuse, rape, family violence, adoption, separation from parents and neglect. For this study the scale was not formally scored, but rather the reporting of any of these items with a high associated perceived level of danger and subsequent impact on everyday life in the past year was classed as a traumatic event. The group was further divided into those experiencing these events before and after age 16 . Within the total sample, 64 participants (16.9\%) had been exposed to traumatic events and $54(14.3 \%)$ before the age of 16 .

\section{Depression}

The Center for Epidemiologic Studies-Depression Scale (CESD) ${ }^{30}$ was used to detect high levels of depressive symptomatology. Depression has been treated as a dichotomous variable with cases being defined as a score above the 16 cut-off point of the CES-D or current treatment with an antidepressant. Of the 378 participants examined, 64 (16.9\%) had CES-D scores of 16 and over and a further 29 participants $(7.7 \%)$ were receiving antidepressant treatment.

\section{Cognitive testing}

COGNITO is a computerised neuropsychological battery, ${ }^{31}$ designed to examine information processing across a wide range of cognitive functions in adults of all ages. Tests are administered using a tactile screen to capture information processing time as well as response accuracy.

Ten cognitive summary variables from the COGNITO battery were considered as dependent variables:

1. Working memory: the simultaneous presentation of auditory and visual attention tasks assessed by subtracting the time taken in milliseconds on this double task from the visual task alone.

2. Narrative recall: total number of correct elements on immediate recall of a story with a temporal progression requiring attention to macrostructure.

3. Description recall: total number of correct elements recalled of a description without thematic progression requiring attention to microstructure and recall of spatial location. The narrative and description recall are similar in terms of word frequency in the language and syntactic structure.

4. Implicit memory: difference in the number of steps in the progressive build-up of names on the screen required for recognition between names never seen and number of names previously learnt in an immediate recall task.

5. Name-face association: number of faces recognised after a delay from a series of 18 faces of which 9 have been previously shown with their corresponding names.

6. Form perception: number of correct answers in the matching of complex forms to a multiple-choice array.

7. Form perception speed: mean time taken in milliseconds for each trial. ${ }^{6}$

8. Phoneme comprehension: number of correct responses in the matching of a word with an image presented as part of a multiple-choice array including semantic, morphological and phonetic distractors.

9. Phoneme comprehension speed: mean time taken in milliseconds to perform. ${ }^{8}$ 
10. Verbal fluency: total sum of the number of words generated in 30 s using both a semantic (vegetables) and phonemic (letter P) cue.

\section{Imaging}

Participants underwent multimodal 3T structural MRI on a single scanner including volumetric T1-weighted scans (176 slices, $1.0 \times 1.0 \mathrm{~mm}, 1.0 \mathrm{~mm}$ slice thickness, $\mathrm{TR}=2300 \mathrm{~ms}$, $\mathrm{TE}=2.98 \mathrm{~ms}$, flip angle $9^{\circ}$ ). Brain tissue segmentation into GM, WM and cerebrospinal fluid was performed using the Gaussian mixture model in VBM toolbox of SPM8 (http://www.fil.ion. ucl.ac.uk/spm). The GM maps were then normalised using the DARTEL algorithm. ${ }^{32}$ Hippocampal region of interests (ROIs) were selected using AAL atlas in MNI space, ${ }^{33} 34$ and then inverse normalised back to each subject's native space using the participant-specific diffeomorphic parameters estimated from the previous DARTEL procedure. The resulting ROIs were also masked using the thresholded GM probability maps (at threshold $\mathrm{p}>0.8$ ) before the total hippocampal volume was calculated.

\section{Statistical analyses}

Cases of depression were first compared with non-cases using a $\mathrm{X}^{2}$ test for categorical variables and Wilcoxon two-sample test for continuous variables. The covariates associated with depression with a $\mathrm{p}<0.10$ (except anxiety and educational level due to collinearity with socioeconomic class) were then entered in a logistic model with the sum of traumatic events.

A linear model adjusted for education, gender and age was used to test the association of continuous cognitive scores with depression or having experienced at least one traumatic event before the age of 16 as well as with hippocampal volume. For two non-continuous cognitive scores (form matching and phoneme comprehension), a logistic model was used after dichotomisation (poor performance being defined as $<7$ and $<9$ correct responses, respectively). A linear model adjusted for intra-cranial volume was also used to test the association of hippocampal volume with depression and having experienced at least one traumatic event. This latter model on traumatic events was further adjusted for education, gender, age and depression. All the analyses were carried out using SAS, V.9.4.

\section{RESULTS}

Trauma and depression

The association between depression and socioeconomic and clinical characteristics is given in table 1 . The range of the total

Table 1 The association between depressive symptomatology (CES-D) and clinical and socioeconomic characteristics

\begin{tabular}{|c|c|c|c|}
\hline & \multicolumn{2}{|l|}{ Depressive symptomatology } & \multirow[b]{3}{*}{ P value* } \\
\hline & CES-D <16 and not treated $(n=285)$ & CES-D $\geq 16$ or treated $(n=93)$ & \\
\hline & n (\%) & n (\%) & \\
\hline Sex, female & $197(69.1)$ & $66(71.0)$ & 0.74 \\
\hline Marital status & & & 0.02 \\
\hline Married or living as a couple & $223(78.3)$ & $61(66.3)$ & \\
\hline Educational level & & & 0.06 \\
\hline College/university & $204(71.8)$ & $57(61.3)$ & \\
\hline Living alone & $37(13.0)$ & $17(18.3)$ & 0.2 \\
\hline Socioeconomic class, $\mathrm{n}=358$ & & & 0.04 \\
\hline Higher managerial, administrative occupations & $75(27.6)$ & $14(16.3)$ & \\
\hline Lower managerial, administrative occupations & $108(39.7)$ & $31(36.0)$ & \\
\hline Intermediate occupations & $31(11.4)$ & $17(19.8)$ & \\
\hline Employers, own account, technical, routine occupation & $30(11.0)$ & $16(18.6)$ & \\
\hline Never worked or long-term unemployed & $28(10.3)$ & $8(9.3)$ & \\
\hline BMI $\left(\mathrm{kg} / \mathrm{m}^{2}\right)$ & & & 0.09 \\
\hline Normal $(<25)$ & $115(40.3)$ & $26(28.0)$ & \\
\hline Overweight (25-30) & $106(37.2)$ & $40(43.0)$ & \\
\hline Obese $(\geq 30)$ & $64(22.5)$ & $27(29.0)$ & \\
\hline Hypertension (treated or SBP >140) & $44(15.4)$ & $22(23.7)$ & 0.07 \\
\hline Cardiovascular disease (declared) & $24(8.4)$ & $10(10.8)$ & 0.5 \\
\hline Diabetes (treated or declared or glycaemia $>7 \mathrm{mmol} / \mathrm{L}$ ), $\mathrm{n}=377$ & $8(2.8)$ & $8(8.6)$ & 0.02 \\
\hline Anxiety symptoms (Spielberger state scale >31) & $71(24.9)$ & $51(54.8)$ & $<0.0001$ \\
\hline Parental history of dementia $(n=377)$ & $151(53.2)$ & $52(55.9)$ & 0.64 \\
\hline Traumatic events at any age (at least 1 event) & $39(13.7)$ & $25(26.9)$ & 0.003 \\
\hline Traumatic events after 16 years only (at least 1 event) & $6(2.1)$ & $4(4.3)$ & 0.25 \\
\hline \multirow[t]{2}{*}{ Traumatic events at 16 years or younger (at least 1 early event) } & $33(11.6)$ & $21(22.6)$ & 0.009 \\
\hline & Median (IQR) & Median (IQR) & P valuet \\
\hline Age (years) & $53(47-56)$ & $53(49-56)$ & 0.76 \\
\hline Hippocampal grey matter volume $\left(\mathrm{mm}^{3}\right)$ & $3689(3525-3951)$ & $3681(3428-3949)$ & 0.66 \\
\hline Intracranial volume $\left(\mathrm{cm}^{3}\right), \mathrm{n}=193$ & $1387(1299-1477)$ & $1354(1268-1451)$ & 0.22 \\
\hline
\end{tabular}

${ }^{*} \mathrm{X}^{2}$ test.

tWilcoxon two-sample test.

$\neq$ With parental history of dementia.

BMI, body mass index; CES-D, Center for Epidemiologic Studies-Depression Scale; SBP, systolic blood pressure. 
Table 2 Adjusted association between childhood trauma and depression $(n=356)$

\begin{tabular}{|c|c|c|c|}
\hline & $\mathrm{OR}^{*}$ & $95 \% \mathrm{Cl}$ & $P$ value \\
\hline Marital status: married or living as a couple & 0.59 & $0.34-1.03$ & 0.06 \\
\hline Socioeconomic class & & & 0.03 \\
\hline Higher managerial, administrative occupations & 1 & & \\
\hline Lower managerial, administrative occupations & 1.57 & $0.76-3.20$ & \\
\hline $\begin{array}{l}\text { Intermediate occupations, employers, own } \\
\text { account, technical, routine occupation }\end{array}$ & 2.94 & $1.40-6.17$ & \\
\hline Never worked or long-term unemployed & 1.56 & $0.57-4.30$ & \\
\hline BMI: overweight or obese & 1.62 & $0.91-2.87$ & 0.1 \\
\hline Hypertension (treated or SBP>140) & 1.19 & $0.62-2.31$ & 0.6 \\
\hline $\begin{array}{l}\text { Diabetes (treated or declared or glycaemia } \\
>7 \mathrm{mmol} / \mathrm{L} \text { ) }\end{array}$ & 2.03 & $0.58-7.18$ & 0.27 \\
\hline Traumatic events before age 16 (at least one) & 2.52 & $1.33-4.80$ & 0.005 \\
\hline
\end{tabular}

${ }^{*}$ Adjusted for other variables in the table.

BMI, body mass index; SBP, systolic blood pressure.

sum of traumatic events was $0-5$ for childhood events and 0-2 for events after 16 years.

Of the 54 participants exposed to childhood trauma, 21 (38.9\%) had a CES-D score of 16 and over or were currently being treated for depression. The association between childhood trauma and depressive symptomatology is significant $(\mathrm{p}=0.009)$. A multivariate logistic model with depression as the dependent variable was used to examine the association between childhoodf traumatic events (at least one) adjusted for marital status, socioeconomic status, hypertension, diabetes and body mass index (table 2). Adjustment was not made for anxiety given the high correlation between performance on the depression and anxiety scales due to overlap in items and also probably high rates of mixed anxiety and depression in participants.

\section{Hippocampal volume}

Structural imaging data were available for the west London centre only ( $\mathrm{n}=193$, see online supplemental table 1 for subsample description). Table 3 shows the associations adjusted forintracranial volume between hippocampal volume as the dependent variable and depression, traumatic events (at least one) before and after the age of 16 . Three linear models are presented showing the independent associations of depression, trauma after 16 years and childhood trauma with hippocampal volume.

In the subsample with an MRI examination, 21 participants were treated with antidepressants. In order to control for any possible effect of treatment on brain volume, we also conducted the analyses excluding participants undergoing treatment. The result did not change and the association with depression adjusted for ICV remained non-significant $(p=0.23)$.

Table 3 Associations between hippocampal volume as the dependent variable and depression or traumatic events (separate linear models)

\begin{tabular}{lcl}
\hline \multirow{2}{*}{ Independent variables } & \multicolumn{2}{l}{$\begin{array}{l}\text { Hippocampal grey matter } \\
\text { volume }\left(\mathrm{mm}^{3}\right)\end{array}$} \\
\cline { 2 - 3 } & $\boldsymbol{\beta}(\mathrm{SE})^{*}$ & P value \\
\hline Depressive symptomatology (CES-D $\geq 16$ or treated) & $44.47(33.65)$ & 0.19 \\
\hline Traumatic events after 16 years (at least oneevent) & $60.25(81.73)$ & 0.46 \\
\hline Traumatic events before 16 years (at least one event) & $-90.52(38.20)$ & 0.02 \\
\hline${ }^{*}$ Adjusted for total intracranial volume. & & \\
CES-D, Center for Epidemiologic Studies-Depression Scale.; &
\end{tabular}

The association with trauma before age 16 remained significant when it was adjusted for ICV, education level, gender and age $(\beta(\mathrm{SE})=-93.4(39.0) ; \mathrm{p}=0.02)$ as well as when depression was further added to the model $(\beta(\mathrm{SE})=-99.4(39.0) ; \mathrm{p}=0.01)$. The relationship with hippocampal volume is not altered with adjustment by depression.

\section{Cognitive performance in middle age}

The association between current cognitive performance based on subscores from COGNITO and traumatic events before age 16 was examined using a multivariate model adjusted by education level, gender and age (table 4).

The number of correct answers for form matching and phoneme comprehension was not statistically associated with traumatic events before 16 years, the adjusted OR (95\% CI) of poor performances was equal to $0.82(0.46-1.47)$ and 0.94 (0.50-1.76), respectively.

A significant association was found between depression and delayed recall of names $(p=0.03)$ but not on the other cognitive measures (online supplemental tables 2 and 3). No significant association was found between hippocampal volume and cognitive measures (online supplemental table 4).

\section{Risk of dementia}

In persons with a family history of dementia, depression prevalence was $25.6 \%$ compared with $23.6 \%$ in those without a family history $(\mathrm{p}=0.64)$. A total of $11.3 \%$ of persons with a family history of dementia reported being exposed to childhood trauma compared with $17.8 \%$ without $(\mathrm{p}=0.07)$.

No interaction effect was found between parental history of dementia and depression in relation to cognition. The interaction for name recall is $\mathrm{p}=0.54$.

Given the complexity of the observed associations we constructed an association graph (figure 1) designed to facilitate understanding and provide a hypothetical basis for future research.

\section{DISCUSSION}

This study aimed to determine whether persons with a history of childhood trauma may be at increased risk of both depression and loss of hippocampal volume in midlife, which may in turn be associated with cognitive performance in persons at increased risk for dementia.

The estimated prevalence of clinically significant depression in this study, based on CES-D scores (16.9\%) and current antidepressant treatment $(7.7 \%)$ was observed to be very high $(24.6 \%)$ compared with $5.1 \%$ estimated for a similar age group in western countries using DSM IV/Composite International Diagnostic Interview criteria. ${ }^{33}$ The rate remains high even if treated depression is excluded on the basis that antidepressants may have in some cases been prescribed for reasons other than depression. Although different measures of depression have been used, previous studies show high concordance between DSM major depression and the CES-D. ${ }^{35}$ While increased rates of depression might have been expected within this cohort enriched for dementia risk, depression prevalence was not observed in this midlife group to be associated with a family history of dementia. One-third of the cohort were exposed to a traumatic event, and of these $18 \%$ experienced the trauma before the age of 16 . We found an association between history of trauma and both clinical-level depression and volume loss in the hippocampus. Lower hippocampal volume was observed to be specifically related to childhood trauma and not with trauma experienced at 


\begin{tabular}{|c|c|c|c|}
\hline \multirow[b]{2}{*}{ Dependent variables } & \multicolumn{3}{|c|}{ Exposure to traumatic event } \\
\hline & $\begin{array}{l}\text { Yes } \\
\text { Mean (SEM)* } \\
\mathrm{N}=54\end{array}$ & $\begin{array}{l}\text { No } \\
\text { Mean (SEM)* } \\
\mathrm{N}=323\end{array}$ & $P$ value \\
\hline Working memory: time difference between double task and simple task (milliseconds) & $-414.3(431.9)$ & $25.2(192.9)$ & 0.33 \\
\hline Form matching: mean time for correct responses (milliseconds) & $5781.9(214.3)$ & $5713.4(95.7)$ & 0.76 \\
\hline Phoneme comprehension: mean time for correct responses (milliseconds) & $1556.7(41.7)$ & $1500.9(18.64)$ & 0.20 \\
\hline Name-face associations: number of names correctly recalled & $4.71(0.28)$ & $5.05(0.13)$ & 0.24 \\
\hline Verbal fluency: number of correct answers & $27.80(0.89)$ & $27.78(0.39)$ & 0.98 \\
\hline Narrative recall: number of correct answers & $12.73(0.61)$ & $12.79(0.27)$ & 0.92 \\
\hline Descriptive recall: number of correct answers & $11.70(0.58)$ & $12.42(0.26)$ & 0.24 \\
\hline Implicit memory: difference between learnt and new items & $1.20(0.10)$ & $1.03(0.05)$ & 0.13 \\
\hline
\end{tabular}

*Adjusted for education level, gender and age.

other ages. This finding is consistent with previous observations that trauma has its greatest effect on brain development through alterations in Hypothalamic-Pituitary-Adrenal (HPA) axis functioning when occurring before full brain maturation. ${ }^{18} 19 \mathrm{It}$ also underscores the need for future research to consider the context and timing of traumatic incidents along developmental trajectories.

In this midlife cohort we observed that severe childhood trauma is associated in midlife with both higher rates of clinical depression and lower hippocampal volume. For those persons who have experienced childhood trauma who develop dementia in the future, it may be postulated that lower volume will add to the burden of hippocampal loss associated with the dementia. We did not at this point, however, observe any association with current cognitive performance or increased dementia risk (family history), suggesting that the direction of causality is probably not persons with a family history of dementia being more vulnerable to early-life trauma.

Investigations of the interaction between adverse childhood experiences with genetic risk factors for AD in regard to laterlife cognitive deterioration have provided mixed findings. ${ }^{22} 36$ A study of childhood abuse and cognition in bipolar disorder has suggested the possibility of a gene-environment interaction implicating genes known to exert a neurotrophic effect in response to cellular injury ${ }^{37}$ notably Apolipoprotein E (ApoE). Polymorphisms on the ApoE gene may thus induce compensatory neurite outgrowth and synaptogenesis following neuronal injury $^{38}$ and thus modulate the neurobiological impact of environmental trauma in children while having an adverse effect in later life. Furthermore HPA axis vulnerability to trauma may further be modulated by genetic and epigenetic factors. 3940 Together these observations suggest a far more complex aetiological model than we have been able to explore here due to lack of statistical power.

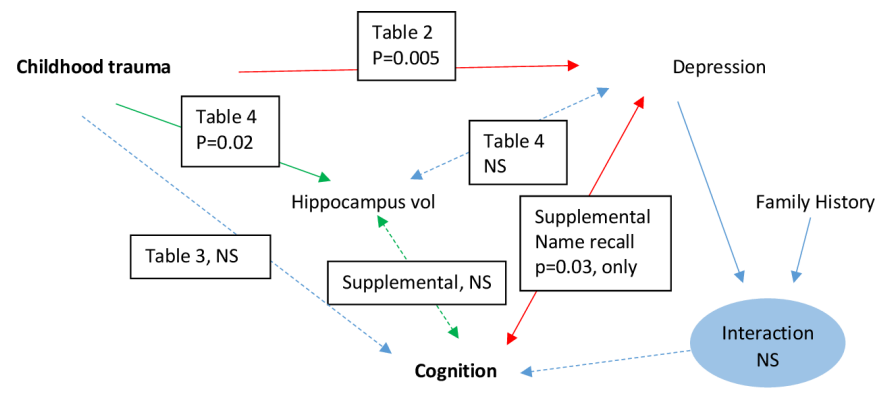

Overall our findings would be consistent with the idea that depression may in some cases be an indicator of underlying HPA axis dysfunction or altered sensitivity due to early-life trauma which has led to associated alterations to the hippocampus. While this cannot be seen in this midlife cohort to be related to either cognitive functioning or dementia risk, hippocampal volume loss may add to the burden of later dementia-related pathology. The aetiology of depression appears in this case to be independent of that of dementia, as previously suggested by O'Brien et $a l^{15}$ in relation to postmortem studies of AD neuropathology in depressed elderly.

Our aetiological model based on cross-sectional data suggests that childhood trauma is associated with both higher rates of depression and lower hippocampal volume. Poorer performance on an episodic memory test is associated with trauma but mediated by depression rather than hippocampal volume. Thus, the relationship of depression to dementia risk in persons experiencing childhood trauma appears to be neither a risk factor nor a prodromal feature of the disease but that of a comorbidity which could potentially add to brain burden.

It is important to note, however, that only $11.3 \%$ of persons at high $\mathrm{AD}$ risk experienced severe early-life trauma. Thus, even if our hypothesis is correct, it will only concern a small proportion of dementia cases. There has been an underlying assumption in most previous studies of depression and AD that depression is a single clinical entity ${ }^{3-6}$ despite well-documented variability in age of onset, precipitating factors, clinical profiles, genetic risk, associated pathologies and treatment response. Our findings do not preclude the possibility of other depression subtypes being linked to AD by alternative mechanisms. The importance of this study lies in the demonstration that depressive symptomatology may be present in persons at high risk for dementia via multiple pathways, and suggest that future studies should consider more carefully the origins of depressive symptomatology when examining its relationship to dementia.

The strengths of the present study are the younger age of the dementia-risk cohort and the identification of a relatively homogeneous depression subgroup. On the other hand the data rely on subjective recall of traumatic events and lack of longitudinal data. Future studies from PREVENT will be able to further explore the hypotheses suggested in this study with a larger cohort, longitudinal data, direct measures of HPA axis functioning and $\mathrm{AD}$ biomarkers with a view to the identification of novel targets for therapeutic intervention in adults with a history of childhood maltreatment at high risk of dementia.

Figure 1 Association graph. NS, non-significant (dotted arrow).

Twitter Craig W Ritchie @Craig_ritchie68 
Acknowledgements The PREVENT Dementia Programme has been financed by a research grant from the UK charity, the Alzheimer's Society and Philanthropic Donations. Genotyping was carried out by Lee Murphy, Edinburgh Clinical Research Facility, University of Edinburgh.

Contributors KR designed the study and wrote the original draft of the manuscript, JTOB, LS, SD, TW, CWR and SG commented on and added to the discussion sections. IC assisted in the design of the study, carried out the statistical analyses and the writing of results.

Funding The Prevent Dementia Study has been funded by the UK Alzheimer Society Grants no. 178, 264.

Competing interests None declared.

Patient consent for publication Not required.

Ethics approval NHS Research Ethics Committee London Camberwell St-Giles (REC reference: 12/LO/1023).

Provenance and peer review Not commissioned; externally peer reviewed.

Data availability statement Data are available upon reasonable request. Data are made available to all researchers subject to approval by the Prevent Dementia Steering Group.

Supplemental material This content has been supplied by the author(s). It has not been vetted by BMJ Publishing Group Limited (BMJ) and may not have been peer-reviewed. Any opinions or recommendations discussed are solely those of the author(s) and are not endorsed by BMJ. BMJ disclaims all liability and responsibility arising from any reliance placed on the content. Where the content includes any translated material, BMJ does not warrant the accuracy and reliability of the translations (including but not limited to local regulations, clinical guidelines, terminology, drug names and drug dosages), and is not responsible for any error and/or omissions arising from translation and adaptation or otherwise.

\section{ORCID iDs}

Karen Ritchie http://orcid.org/0000-0002-0688-8982

John T O'Brien http://orcid.org/0000-0002-0837-5080

\section{REFERENCES}

1 Kral VA. The relationship between senile dementia (Alzheimer type) and depression. Can J Psychiatry 1983;28:304-6.

2 Engedal K, Barca ML, Laks J, et al. Depression in Alzheimer's disease: specificity of depressive symptoms using three different clinical criteria. Int I Geriatr Psychiatry 2011;26:944-51.

3 Lyketsos CG, Olin J. Depression in Alzheimer's disease: overview and treatment. Biol Psychiatry 2002;52:243-52.

4 Starkstein SE, Jorge R, Mizrahi R, et al. The construct of minor and major depression in Alzheimer's disease. Am J Psychiatry 2005;162:2086-93.

5 Mourao RJ, Mansur G, Malloy-Diniz LF, et al. Depressive symptoms increase the risk of progression to dementia in subjects with mild cognitive impairment: systematic review and meta-analysis. Int J Geriatr Psychiatry 2016;31:905-11.

6 Lebedev AV, Beyer MK, Fritze F, et al. Cortical changes associated with depression and antidepressant use in Alzheimer and Lewy body dementia: an MRI surface-based morphometric study. Am J Geriatr Psychiatry 2014;22:4-13.

7 O'Brien J, Perry R, Barber R, et al. The association between white matter lesions on magnetic resonance imaging and noncognitive symptoms. Ann N Y Acad Sci 2000:903:482-9.

8 Tagariello P, Girardi P, Amore M. Depression and apathy in dementia: same syndrome or different constructs? A critical review. Arch Gerontol Geriatr 2009;49:246-9.

9 Qiu WQ, Sun X, Selkoe DJ, et al. Depression is associated with low plasma Abeta42 independently of cardiovascular disease in the homebound elderly. Int I Geriatr Psychiatry 2007;22:536-42.

10 Namekawa Y, Baba $\mathrm{H}$, Maeshima $\mathrm{H}$, et al. Heterogeneity of elderly depression: increased risk of Alzheimer's disease and A $\beta$ protein metabolism. Prog Neuropsychopharmacol Biol Psychiatry 2013;43:203-8.

11 Sun X, Steffens DC, Au R, et al. Amyloid-Associated depression. Arch Gen Psychiatry 2008:65:542-50.

12 Chi S, Yu J-T, Tan M-S, et al. Depression in Alzheimer's disease: epidemiology, mechanisms, and management. J Alzheimers Dis 2014;42:739-55.

13 Ringman JM, Liang L-J, Zhou Y, et al. Early behavioural changes in familial Alzheimer's disease in the dominantly inherited Alzheimer network. Brain 2015;138:1036-45.
14 Ownby RL, Crocco E, Acevedo A, et al. Depression and risk for Alzheimer disease: systematic review, meta-analysis, and metaregression analysis. Arch Gen Psychiatry 2006;63:530-8.

15 O'Brien J, Thomas A, Ballard C, et al. Cognitive impairment in depression is not associated with neuropathologic evidence of increased vascular or Alzheimer-type pathology. Biol Psychiatry 2001;49:130-6.

16 Ritchie K, Carrière I, Berr C, et al. The clinical picture of Alzheimer's disease in the decade before diagnosis: clinical and biomarker trajectories. J Clin Psychiatry 2016;77:e305-11.

17 Malhi GS, Parker GB, Greenwood J. Structural and functional models of depression: from sub-types to substrates. Acta Psychiatr Scand 2005;111:94-105.

18 Teicher MH, Andersen SL, Polcari A, et al. Developmental neurobiology of childhood stress and trauma. Psychiatr Clin North Am 2002;25:397-426.

19 Heim C, Nemeroff CB. The role of childhood trauma in the neurobiology of mood and anxiety disorders: preclinical and clinical studies. Biol Psychiatry 2001;49:1023-39.

20 Bernet CZ, Stein MB. Relationship of childhood maltreatment to the onset and course of major depression in adulthood. Depress Anxiety 1999;9:169-74.

21 Kaufman J, Charney D. Effects of early stress on brain structure and function: implications for understanding the relationship between child maltreatment and depression. Dev Psychopathol 2001:13:451-71.

22 Ritchie K, Jaussent I, Stewart R, et al. Adverse childhood environment and late-life cognitive functioning. Int I Geriatr Psychiatry 2011;26:503-10.

23 Navalta CP, Polcari A, Webster DM, et al. Effects of childhood sexual abuse on neuropsychological and cognitive function in college women. J Neuropsychiatry Clin Neurosci 2006:18:45-53.

24 Bremner JD, Vermetten E, Afzal N, et al. Deficits in verbal declarative memory function in women with childhood sexual abuse-related posttraumatic stress disorder. J Nerv Ment Dis 2004;192:643-9.

25 Bremner JD, Randall P, Scott TM, et al. Deficits in short-term memory in adult survivors of childhood abuse. Psychiatry Res 1995;59:97-107.

26 McKeever A, Paris AF, Cullen J, et al. Hippocampal Subfield volumes in middle-aged adults at risk of dementia. J Alzheimers Dis 2020;75:1211-8.

27 Ritchie $C W$, Ritchie K. The prevent study: a prospective cohort study to identify mid-life biomarkers of late-onset Alzheimer's disease. BMJ Open 2012:2:e001893.

28 Wolfe J, Kimerling R. Gender issues in the measurement of post-traumatic stress disorder. In: Wilson JP, Keane TM, eds. Assessing psychological trauma and PTSD. New York: Guilford Press, 1997

29 American Psychiatric Association. Diagnostic and statistical manual of mental disorders. Washington: American Psychiatric Association, 2000.

30 Radloff LS: the CES-D scale: a self-report depression scale for research in the general population. Appl Psychol Measurement 1977;1:385-401.

31 Ritchie K, de Roquefeuil G, Ritchie CW, et al. COGNITO: computerized assessment of ageing-related changes in cognitive processing. J Psychol Psychother 2014.

32 Ashburner J. A fast diffeomorphic image registration algorithm. Neuroimage 2007;38:95-113

33 Tzourio-Mazoyer N, Landeau B, Papathanassiou D, et al. Automated anatomical labeling of activations in SPM using a macroscopic anatomical parcellation of the MN MRI single-subject brain. Neuroimage 2002;15:273-89.

34 Kessler RC, Birnbaum HG, Shahly V, et al. Age differences in the prevalence and comorbidity of DSM-IV major depressive episodes: results from the who world mental health survey initiative. Depress Anxiety 2010;27:351-64.

35 Sjöberg L, Karlsson B, Atti A-R, et al. Prevalence of depression: comparisons of different depression definitions in population-based samples of older adults. J Affect Disord 2017:221:123-31.

36 Moceri VM, Kukull WA, Emanual I, et al. Using census data and birth certificates to reconstruct the early-life socioeconomic environment and the relation to the development of Alzheimer's disease. Epidemiology 2001;12:383-9.

37 Savitz J, van der Merwe L, Stein DJ, et al. Genotype and childhood sexual trauma moderate neurocognitive performance: a possible role for brain-derived neurotrophic factor and apolipoprotein E variants. Biol Psychiatry 2007;62:391-9.

38 Poirier J. Apolipoprotein E in animal models of CNS injury and in Alzheimer's disease. Trends Neurosci 1994;17:525-30.

39 Ancelin M-L, Scali J, Norton J, et al. The effect of an adverse psychological environment on salivary cortisol levels in the elderly differs by 5-HTTLPR genotype. Neurobiol Stress 2017;7:38-46.

40 Wrigglesworth J, Ancelin M-L, Ritchie K, et al. Association between DNA methylation of the KITLG gene and cortisol levels under stress: a replication study. Stress 2019;22:162-8. 